\title{
Unexpected borderline malignant and malignant smooth muscle cell tumors of the uterine corpus in women treated with LH-RH analogues
}

\author{
Carla C.A.P. Wauters*a, Bram W.A. Ter Harmsel ${ }^{\mathrm{b}}$, Marinus P.M. Hermans ${ }^{\mathrm{b}}$, Frank Smedts ${ }^{\mathrm{c}}$ \\ "Department of Pathology, Diagnostic Centre SSDZ, Delft, The Netherlands \\ ${ }^{b}$ Department of Obstetric's and Gynecology, Reinier de Graaf Hospital, Delft, The Netherlands \\ 'Department of Pathology, Canisius-Wilhelmina Hospital, Nijmegen, The Netherlands
}

Received 8 March 1995; revision received 19 May 1995; accepted 14 June 1995

\begin{abstract}
In this report, two cases of uterine smooth muscle cell tumors, one of uncertain malignant potential and one clearly malignant, are described in women treated for prolonged periods with luteinizing hormone-releasing hormone (LH-RH) analogues. Due to lengthy monitoring of LH-RH therapy, surgical intervention and histologic classification of these tumors was late in the course of disease, resulting in a delay in definite treatment. The risk to these women is discussed. The effects of LH-RH analogue therapy on fibroids is reviewed. Suggestions are put forward for monitoring LH-RH analogue therapy.
\end{abstract}

Keywords: LH-RH agonist; Smooth muscle tumor of uncertain malignant potential; Leiomyosarcoma

\section{Introduction}

As tumor volume in both benign and malignant smooth muscle cell tumors of the uterine corpus has been shown to be oestrogen dependent, much research has been directed toward growth modification of these tumors with hormones. In recent years there has been increased interest in the application of luteinizing hormone-releasing hormone (LH-RH) agonists. These induce suppression of ovarian hormonal function and a hypoestrogenic state, inducing a reduction in fibroid volume which may exceed $50 \%$ [6]. The exact mechanism by which volume reduction is attained is at present unknown. However, this therapeutic approach is widely used. A drawback in treating fibroids with LH-RH analogues for long periods is that, in women who do not respond to therapy, there will considerable delay before surgical intervention and subsequent histologic evaluation of the uterine neoplasms. In the case of a leiomyofibroma, the delay will have no adverse effect

\footnotetext{
* Corresponding author, Department of Pathology Erasmus University Rotterdam, dr. Molenwaterplein 50, P.O. Box 1738, 3000 DR Rotterdam, The Netherlands. Tel.: + 31 104087901; Fax: + 31 104366660 .
}

for the patient. However, if the smooth muscle cell tumor is of uncertain malignant potential or malignant, the delay may have severe consequences.

Two cases are illustrated in which treatment of uterine fibroids with LH-RH analogues led to a considerable delay in histologic classification and possibly exposed patients to risk of which the severity is difficult to estimate.

\section{Case reports}

\subsection{Case 1}

A 33-year-old primigravida with an uneventful past medical history presented with excessive menstrual bleeding while on sequential hormonal contraceptives. On physical examination, her uterus was the size of a 14-week pregnancy. Ultrasonographic examination revealed a solid subserosal mass in the posterior wall of the uterus, the diameter was $9.8 \mathrm{~cm}$. An endometrial curetting was normal.

As the patient wished to preserve her fertility, she was treated for 6 months with an LH-RH analogue (Decapeptyl $\mathbb{R}$ Ferring, Haarlem, The Netherlands). The diameter of the uterine mass decreased to $4.9 \mathrm{~cm}$. She 
was seen at regular intervals for 1 year after completion of the treatment. During this period she did not become pregnant. Ultrasonography performed at the end of this period revealed that the solid mass had increased in diameter and was $8.9 \mathrm{~cm}$. Surgical removal was decided on.

Three masses were enucleated; one was $0.9 \mathrm{~cm}$, one was $2.5 \mathrm{~cm}$ and the third was $9 \mathrm{~cm}$ in diameter and weighed $330 \mathrm{~g}$. All tumors had the same macroscopic features and firm rubbery consistency. From each tumor, excisions were taken at intervals of approximately $1 \mathrm{~cm}$ and routine $\mathrm{H} \& E$ stained sections were prepared. Microscopic examination revealed three tumors composed of whirling bundles of spindle-shaped smooth muscle cells. Sections from the largest myoma (Fig. 1), however, were different when compared with the smaller tumors in that there was moderate nuclear atypia and an average of 8 mitotic figures were counted in 10 consecutive fields (magnification, $400 \times)$. The diagnosis of a smooth muscle tumor of uncertain malignant potential was rendered.

Further treatment consisted of a hysterectomy. No residual tumor was found in the uterine specimen. The patient is alive and well, 26 months after hysterectomy, with no evidence of disease.

\subsection{Case 2}

A 47-year-old woman, gravida 2, para 2, presented with severe blood loss leading to anaemia, requiring blood transfusions. On physical examination, the uterus was the size of a 10 -week pregnancy. No additional tests were performed. For the following 4

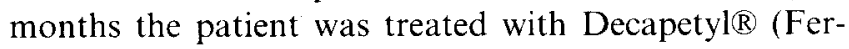
ring Haarlem, The Netherlands). During therapy, uterine size decreased but the hypermenorrhoe recurred on cessation of hormonal treatment. Surgical intervention was decided on.

The resected uterus was deformed and weighed $800 \mathrm{~g}$. Dissection revealed several well-delineated firm white-grey tumors between 1 and $7 \mathrm{~cm}$ in diameter and one less well delineated grey mass with a diameter of $4.5 \mathrm{~cm}$ and a soft putty-like consistency. Histologic examination of the former nodules showed features consistent with leiomyomas. The latter tumor was hypercellular and composed of highly pleomorphic cells with a variable amount of eosinophilic cytoplasma and large polymorphic nuclei. Dispersed bizarre giant cells were also seen. A maximum of 14 mitotic figures in 10 consecutive fields (magnification, $400 \times$ ) were counted (Fig. 2). There was no evidence of vascular invasion. A pleomorphic leiomyosarcoma was diagnosed.

Further treatment consisted of abdominal radiotherapy. The patient is alive and well, 23 months after hysterectomy, with no evidence of disease.

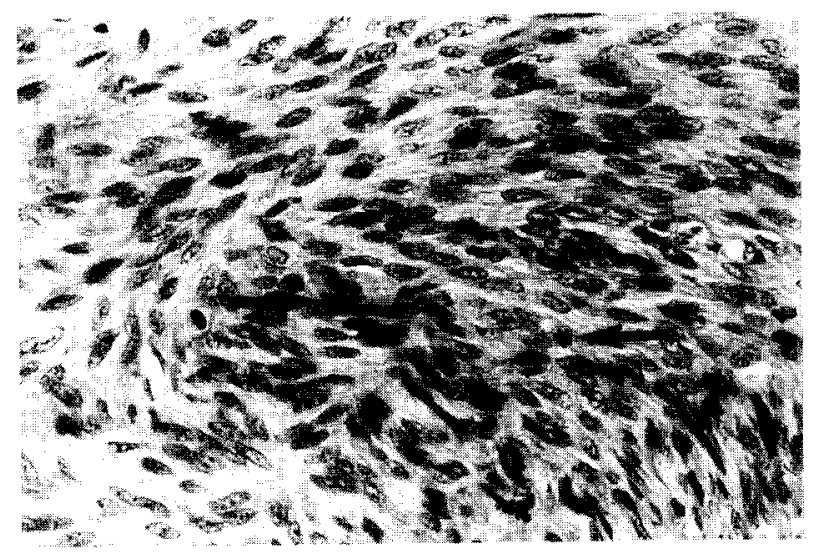

Fig. 1. Case 1: smooth muscle cell tumor composed of elongated cells with uniform nuclei and eosinophilic cytoplasm. Several mitotic figures (arrows) are visible. $400 \times, \mathrm{H} \& \mathrm{E}$.

\section{Discussion}

During the past 10 years, treatment of uterine leiomyomas with LH-RH analogues has become extensive $[1-5]$. These analogues are derivatives of the hypothalamic hormone LH-RH in which substitutions in the amino acid structure have made the analogue 40-200 times more potent than naturally occuring LH-RH. The mechanism of their action is complex; however, they are thought to bind pituitary receptors, resulting in suppression of the pituitary-ovarian axis and inducing a state of pseudo-menopause. The resultant hypoestrogenemic state induces myoma regression. Furthermore, it is thought that LH-RH analogues have a direct inhibitory effect on smooth muscle cell growth [2]. During therapy, significant shrinkage is seen in most fibroids; smaller fibroids have even been shown to regress completely. On cessation of therapy, regrowth of fibroids occurs in premenopausal women. For this reason, LH-RH analogue therapy is given prior to operative therapy. The most important advantage of

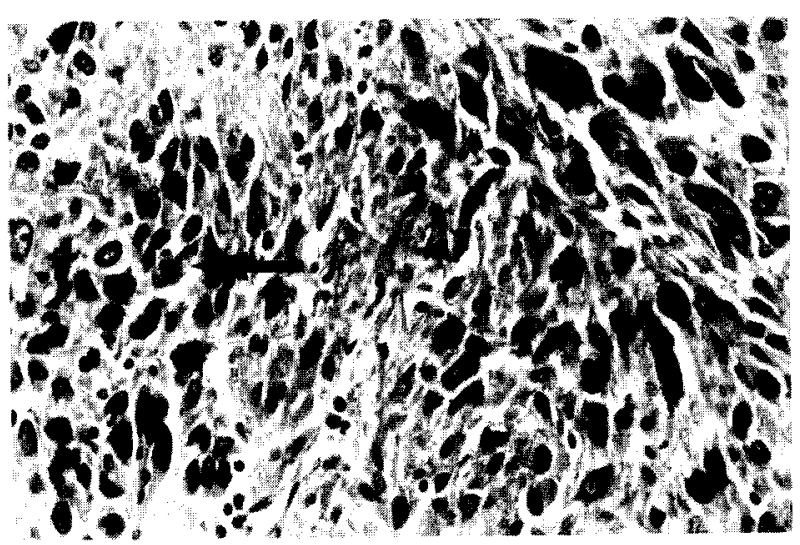

Fig. 2. Case 2: lciomyosarcoma. This neoplasm composed of highly atypical smooth muscle cells with many bizarre giant cells and scattered mitolic figures (arrow). $400 \times . \mathrm{H} \& \mathrm{E}$ 
this approach is that there is considerably less blood loss during surgery. Studies demonstrate that postoperative fertility is improved after myomectomy in patients previously treated with LH-RH analogues [3]. In perimenopausal women, surgery may be avoided due to the fact that the volume reduction of the myoma will sufficiently alleviate symptoms from premenopause up to menopause, after which myomas usually regress spontaneously.

In the two cases described, we demonstrate that this therapeutic approach may also have a number of disadvantages. Malignant and borderline malignant smooth muscle cell tumors causing symptoms may also be treated with LH-RH analogues prior to myomectomy or hysterectomy. This will cause considerable delay between diagnosis and histologic examination of these myomas. The question is whether this approach entails any danger for the patient. In general, only very small numbers of myomas are potentially malignant or clearly malignant. Diagnosing these tumors at a later date should not offset the net beneficial effect of this valuable therapeutic approach. In the case of a leiomyoma of borderline malignancy, it is not clear what effect delay in surgical intervention will have. In these tumors metastasis have not been described and their biological behaviour is not known [3].

In leiomyosarcoma, delay in diagnosis could be expected to have a significant effect on patient outcome as disease may spread in the period during which the LH-RH analogue is given. However, very little is known regarding the metastatic potential of a leimyosarcoma and if LH-RH analogue treatment has any effect on metastatic potential [7].

It has been reported that gonadotropin-releasing hormones induce significant volume reductions in both benign and malignant smooth muscle cell tumors of the uterine corpus. Quantitative morphologic evaluation of these tumors reveals a significant reduction in cellularity; however, fibrosis, edema or mitotic activity are not influenced. This implies that the same histological criteria may be applied to uterine smooth muscle tumors in women treated with LH-RH analogues as are applied to tumors in untreated patients $[6,8]$.

There is no clinical parameter capable of indicating the possible malignant potential of a smooth muscle tumor at the beginning of the hormonal treatment [9].

Taking a thick needle biopsy prior to treatment is not recommended due to sampling error associated with this approach.

In summary, we feel that a careful clinical evaluation of the patient is necessary during hormonal therapy; this involves following both subjective and objective symptoms as well as tumor size. In case of severe abdominal pain, persistent vaginal bleeding, increase in tumor size or no objective therapy response, short term surgical intervention should be seriously considered.

\section{Acknowledgments}

The authors thank M.W. Snieders, pathologist, Department of Pathology at the St. Ignatius Hospital, Breda, and A. Logmans gynaecologist, Department of Gynaecology, Dr. Daniel de Hoed Hospital, Rotterdam, for contributing the second case.

\section{References}

[1] Friedman AJ, Lobel SM, Mitchell SR, Barbieri RL. Efficacy and safety considerations in women with uterine leiomyomas treated with gonadotropin-releasing hormone agonist: The estragon threshold hypothesis. Am J Obstet Gynecol 1990; 163: 11141119.

[2] Kettel LM, Murphy AA, Morales AJ, River J, Vale W, Yen SS. Rapid regression of uterine leiomyomas in response to daily administration of gonadotropin-releasing hormone antagonist. Fertil Steril 1993; 60: 642-646.

[3] Vollenhoven BJ, Lawrence AS, Healy DL. Uterine fibroids: a clinical review. Br J Obstet Gynecol 1990; 75: 529-531.

[4] Leusden HA. Symptom-free interval after triptorelin treatment of uterine fibroids: long-term results. Gynecol Endocrinol 1992; 2: $189-198$.

[5] Thompson JD, Brich HW. Indications for hysterectomy. Clin Obstet Gynecol 1981; 24: 1245-1258.

[6] Hendrikson MR, Kempson RL. Surgical pathology of the uterine corpus. Philadelphia, London: WB Saunders Company, 1980; 12/18: 468-529.

[7] Leibsohn S, d'Ablaing G, Mishell DR, Schlaerth JB. Leiomyosarcoma in a series of hysterectomies performed for presumed uterine leiomyomas. Am J Obstest Gynecol 1990; 162: 968-976.

[8] Upadhyaya NB, Doody MC, Googe PB. Histopathological changes in leiomyomata treated with leuprolide acetate. Fertil Steril 1990; 54: 811-814.

[9] Schwartz LS, Diamond MP, Schwartz PE. Leiomyosarcomas: Clinical presentation. Am J Obstet Gynecol 1993; 168: 180-183. 